\title{
Change of the emission spectra in organic light-emitting diodes by layer thickness modification
}

\author{
C. H. Cheung and A. B. Djurišića) \\ Department of Physics, University of Hong Kong, Pokfulam Road, Hong Kong \\ C. Y. Kwong \\ Department of Electrical \& Electronic Engineering, University of Hong Kong, \\ Pokfulam Road, Hong Kong \\ H. L. Tam and K. W. Cheah \\ Department of Physics, Hong Kong Baptist University, Kowloon Tong, Hong Kong \\ Z. T. Liu \\ Department of Physics, University of Hong Kong, Pokfulam Road, Hong Kong \\ W. K. Chan \\ Department of Chemistry, University of Hong Kong, Pokfulam Road, Hong Kong \\ P. C. Chui \\ Department of Electrical \& Electronic Engineering, University of Hong Kong, \\ Pokfulam Road, Hong Kong \\ J. Chan and A. D. Rakić \\ School of Information Technology and Electrical Engineering, The University of Queensland, \\ Brisbane Qld 4072, Australia
}

(Received 14 April 2004; accepted 12 August 2004)

\begin{abstract}
Electroluminescence and photoluminescence of organic light-emitting diodes consisting of an indium tin oxide anode, $\mathrm{N}, \mathrm{N}^{\prime}$-di(naphthalene-1-yl)-N, $\mathrm{N}^{\prime}$-diphenyl-benzidine as a hole transport layer, tris (8-hydroxyquinoline) aluminum as emitting layer, and an $\mathrm{Ag}$ cathode were measured for different layer thickness values. It was found that, for a certain range of thickness values, multiple peak emission can be achieved. In addition, the emission spectra were dependent on the viewing angle. For the optimized thickness values, normal incidence chromaticity coordinates achieved were 0.32 and 0.43. Possible explanations for observed unexpected behavior are discussed. (C) 2004 American Institute of Physics. [DOI: 10.1063/1.1802386]
\end{abstract}

Tris (8-hydroxyquinoline) aluminum $\left(\mathrm{Alq}_{3}\right)$ is a material that is very commonly used in organic light-emitting diodes (OLEDs), either as an electron transport or emitting layer, having a broad green emission. ${ }^{1,2} \mathrm{~N}, \mathrm{~N}^{\prime}$-di(naphthalene-1yl)-N, $\mathrm{N}^{\prime}$-diphenylbenzidine (NPB) is a commonly used hole transport material, which can also be used for realization of blue light-emitting devices. ${ }^{3,4}$ OLEDs based on NPB and/or $\mathrm{Alq}_{3}$ have been extensively studied. While some interference ${ }^{5}$ and weak microcavity ${ }^{6,7}$ effects have been previously reported, it is generally considered that a simple $\mathrm{NPB} / \mathrm{Alq}_{3}$ device would give a single peak emission that would exhibit small, if any, blueshift with the viewing angle. The multiple peak emission can be achieved from a single emitting layer using microcavity structures. ${ }^{8,9}$ However, a microcavity OLED typically exhibits a significant blueshift with the viewing angle. ${ }^{9-11}$

In this work, we demonstrate that a multipeak emission can be achieved from a conventional, two-layer OLED consisting of an indium tin oxide (ITO) anode, a layer of NPB as a hole transport layer, a layer of $\mathrm{Alq}_{3}$ as electron transport/ emitting layer, and a top silver cathode $(\sim 70 \mathrm{~nm})$. The NPB and $\mathrm{Alq}_{3}$ (from H. W. Sands) were purified by sublimation before device fabrication, and microcavity OLEDs were fabricated by evaporation in a high vacuum $\left(\sim 10^{-6}\right.$ Torr $)$. The

\footnotetext{
a)Electronic mail: dalek@hkusua.hku.hk
}

layer thickness during deposition was given by a quartz thickness monitor, and verified after deposition by a step profiler. A HeCd laser (325 nm) and Xe lamp with a monochromator $(420 \mathrm{~nm})$ were used as excitation sources for photoluminescence (PL), while for electroluminescence (EL) a Keithley 2400 source meter was used to bias the devices. The spectra were recorded using fiberoptic spectrometer PDA-512-USB (Control Development Inc.).

Figure 1 shows the normal incidence EL spectra for different $\mathrm{NPB} / \mathrm{Alq}_{3}$ thickness values. It can be observed that the emission spectrum is strongly dependent on the device thickness, as well as the position of the $\mathrm{NPB} / \mathrm{Alq}_{3}$ interface for the same total device thickness. In some cases, up to three peaks can be observed (devices with 59/125 and $59 / 139 \mathrm{NPB} / \mathrm{Alq}_{3}$ thickness in nanometers). For large device thickness, the emission spectrum becomes very broad. Two-peak emission as a consequence of wide-angle interference was reported by So et al. ${ }^{5}$ The important difference between the results presented here and their work is that our devices contain no additional $\mathrm{SiO}_{2}$ layer and that we observed the multiple peak emissions for a much wider range of thickness values. Existing theory on the emission spectra of the microcavity devices ${ }^{8,9}$ can be easily modified to simulate the spectra of an ordinary OLED by treating the ITO layer as a mirror. While the simulated spectra demonstrate that it is indeed possible to obtain two-peak emission for suitably chosen NPB and $\mathrm{Alq}_{3}$ thicknesses, the agreement is 

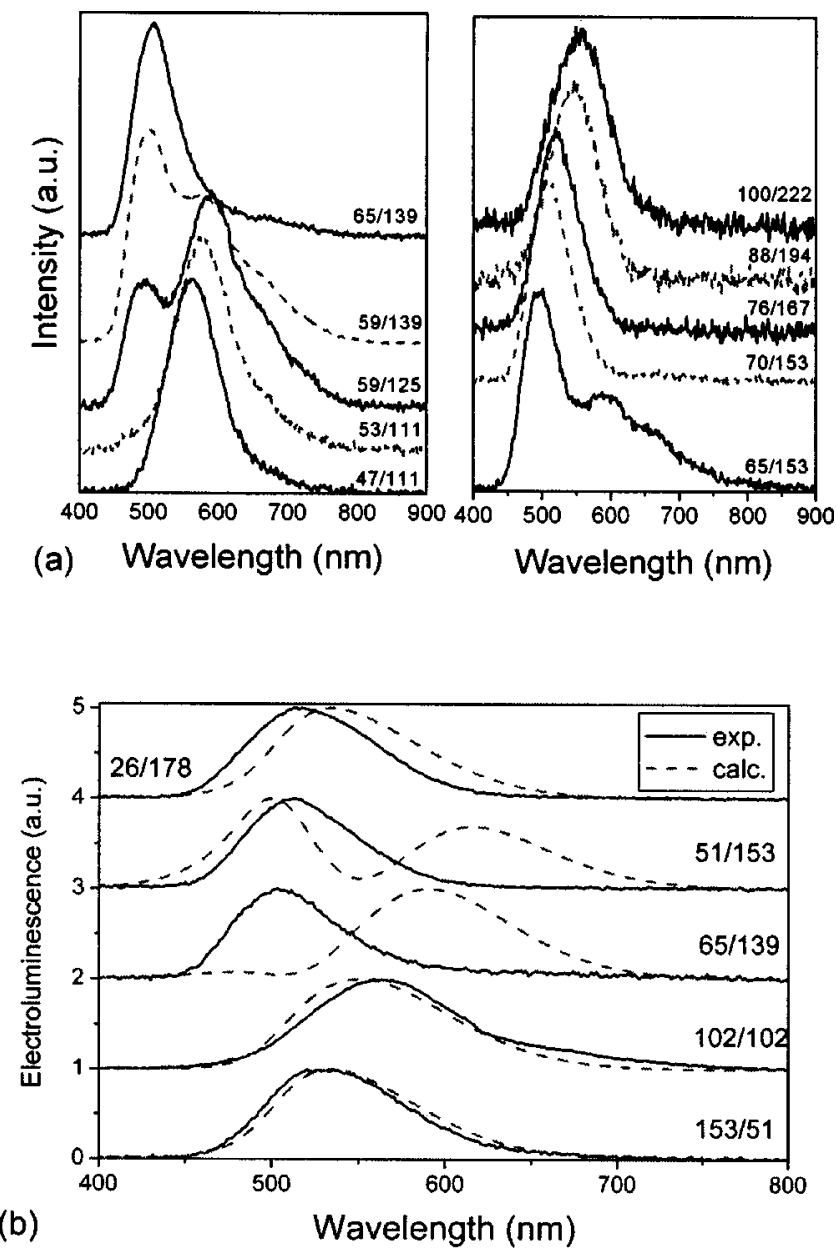

FIG. 1. (a) EL spectra of OLEDs for different thicknesses of NPB and $\mathrm{Alq}_{3}$ layers. (b) EL spectra of OLEDs for different positions of $\mathrm{NPB} / \mathrm{Alq}_{3}$ interface. Solid line denotes experimental data, while dashed line denotes calculated spectra. All the spectra were measured at $0^{\circ}$ viewing angle, and the layer thickness is given in nanometers.

not equally good for all the devices. In addition, we observed significant differences between the EL and PL spectra, indicating that simple interference phenomena are likely not a sufficient explanation for the observed experimental results.

In order to investigate the origin of the observed features in more detail, we have measured the EL spectra at different viewing angles. A behavior significantly different from expected small blueshift was found. Figure 2 shows the EL spectra of the 65/153 device for different viewing angles. The inset shows the chromaticity coordinates. At $0^{\circ}$ viewing angle, the device exhibits blue-white emission, while at larger viewing angles the device exhibits yellow-white emission. The surface-normal emission shows two peaks, one blue-green and one orange. The peaks do not show a significant shift with the viewing angle. However, the ratio of the peak intensities significantly changes with the viewing angle. At smaller angles the higher energy peak is the dominant one, while the opposite is the case at larger angles. Such a behavior is unexpected, considering that the previous works demonstrated that a noncavity OLED would show no significant change in the emission spectra with the viewing angle. Another unexpected feature is the difference between the angular dependence of the EL and PL spectra, especially when the wavelength is chosen in such a manner that only $\mathrm{Alq}_{3}$ is excited. Some difference between the EL and PL spectra is

Downloaded 08 Nov 2006 to 147.8.21.97. Redistribution subject

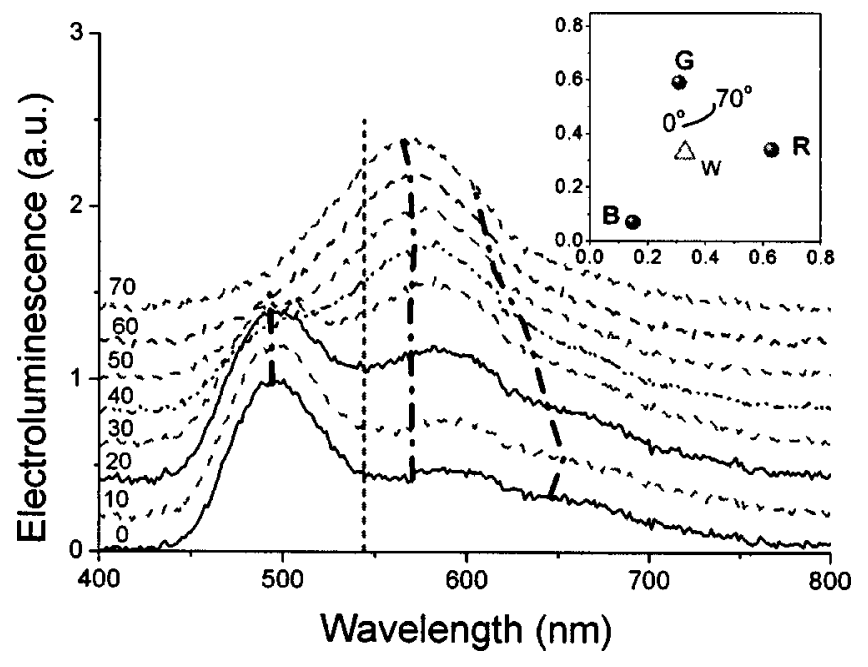

FIG. 2. EL spectra of a NPB $(65 \mathrm{~nm}) / \mathrm{Alq}_{3}(153 \mathrm{~nm})$ OLED for different viewing angles. The dash-dot lines indicate peak positions as obtained in the fitting process. The position of the PL from an $\mathrm{Alq}_{3}$ film is also shown (dashed line). The inset shows chromaticity coordinates corresponding to the EL spectra.

expected due to the different positions of the emission region for electrical and optical excitation. However, the interference phenomena as suggested by So et al., ${ }^{5}$ do not provide sufficient explanation of the measured spectra. It should also be noted that the EL spectra do not show significant dependence on the driving voltage, which would be expected if there were significant shifts in the location of the recombination zone.

The obtained PL spectra for different viewing angles are shown in Fig. 3(a) (excitation wavelength $325 \mathrm{~nm}$ ) and 3(b) (excitation wavelength $420 \mathrm{~nm}$ ). For $325 \mathrm{~nm}$ excitation, we can observe the spectrum which strongly resembles a superposition of a strong NPB and weak $\mathrm{Alq}_{3}$ PL. When we excite the structure with a $420 \mathrm{~nm}$ light, we can see a small redshift of the PL peak upon increasing the viewing angle. While the $\mathrm{PL}$ spectra of $\mathrm{NPB} / \mathrm{Alq}_{3}$ film on a quartz substrate show $\mathrm{PL}$ from both NPB and $\mathrm{Alq}_{3}$ similar to the PL of the OLED device, the intensity ratios of the two peaks are different (PL from $\mathrm{Alq}_{3}$ is stronger from the film). For $420 \mathrm{~nm}$ excitation of the $\mathrm{NPB} / \mathrm{Alq}_{3}$ film, no significant shift with the viewing angle can be observed, and the shoulder at $\sim 460 \mathrm{~nm}$, which can be observed in the PL of an OLED, is absent in the PL of the film. PL spectra at both wavelengths are significantly different from the EL spectra. It is known that the different EL and PL spectra can be obtained from the blends of organic materials due to formation of exciplex complexes. ${ }^{12,13}$ However, an exciplex emission should not exhibit any significant variation with the viewing angle. Interference effects hypothesis does not provide sufficient explanation for the differences between the EL and PL spectra and their behavior with respect to the viewing angle, although it is possible that the agreement between the calculated and experimental data could be improved by better estimate of the emission region position and width, as well as different emitting dipole density distribution in the case of optical excitation.

In order to explain the origin of the unusual behavior observed, we have fitted the EL spectra shown in Fig. 2 with the sum of three Gaussian peaks for each of the viewing angles. The peak positions for different viewing angles are also shown in Fig. 2. The position of the PL from the $\mathrm{Alq}_{3}$ AIP license or copyright, see http://apl.aip.org/apl/copyright.jsp 

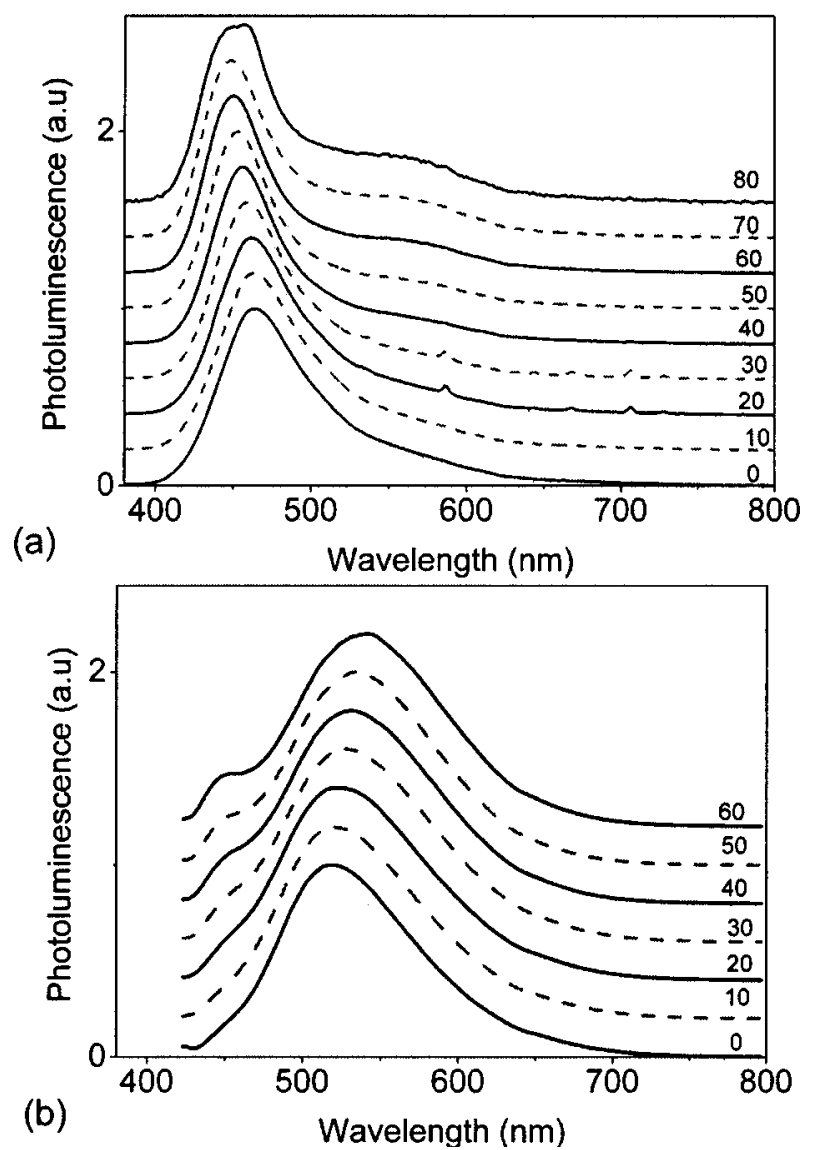

FIG. 3. (a) PL of a NPB $(65 \mathrm{~nm}) / \mathrm{Alq}_{3}(153 \mathrm{~nm})$ OLED for different viewing angles at $325 \mathrm{~nm}$ excitation; and (b) PL of a $\mathrm{NPB}(65 \mathrm{~nm}) / \mathrm{Alq}_{3}(153 \mathrm{~nm})$ OLED for different viewing angles at $420 \mathrm{~nm}$ excitation.

film is also indicated. The PL peak from NPB is $\sim 2.75 \mathrm{eV}(\sim 450 \mathrm{~nm})$. It can be observed that the positions of the two lower wavelength (higher energy) features do not change significantly with the viewing angle, and the weak red emission shows a blueshift with the increasing viewing angle. The shift of the chromaticity coordinates is due mainly to the decreasing intensity of the blue-green emission. It was proposed that the $\mathrm{PL}$ of $\mathrm{Alq}_{3}$ originates from three different levels. ${ }^{14,15}$ The proposed energies of these transitions were $2.45,2.30$, and $2.04 \mathrm{eV}(506,539$, and $608 \mathrm{~nm}),{ }^{14}$ or 2.62 , 2.60 , and $2.34 \mathrm{eV}(473,477$, and $530 \mathrm{~nm}){ }^{15}$ The peak positions obtained in this work are closer to those proposed by Curry and Gillin, ${ }^{14}$ although our peak positions do not exactly match those proposed in their work.

It should be noted that in both previous reports ${ }^{14,15}$ the energy levels were deduced by fitting, while here, two emission peaks are clearly resolved. Moreover, the variation in the relative peak intensity with the viewing angle can be clearly observed (at smaller angles the $\sim 495 \mathrm{~nm}$ peak is dominant, at larger angles the $\sim 570 \mathrm{~nm}$ peak is dominant). There are two possible causes for this: interference phenomena or some kind of an energy transfer between two or three (weak feature between 1.9 and $2.0 \mathrm{eV}$ ) different levels. In either case, observed phenomena are related to the weak microcavity effects in the device, since multiple peaks are not observed in the emission from the edge of the substrate. We have already shown that the theoretical simulations do not show good agreement with the emission spectra for all de- vices. Increased discrepancy between the calculated and experimental spectra for larger device thickness can also be observed in other reported works in the literature. ${ }^{5,7}$ An alternative explanation could possibly be associated with the polariton emission due to coupling between the photon and the exciton mode. Polariton emission has, up to date, been demonstrated only in PL from organic microcavities containing relatively narrow emitting material. ${ }^{16}$ It requires large oscillator strength, and matching of the widths of the photon and exciton modes. The estimated widths of the photon mode $(\sim 100 \mathrm{~nm})$ and $\mathrm{Alq}_{3}$ absorption peak $(\sim 65 \mathrm{~nm})$ are similar. Device thickness, which is larger than usual in OLEDs, may account for the fact that this effect has been previously unobserved. The change of the peak intensities with the viewing angle and thus the longitudinal wave vector bears similarity to polariton emission. Since the phenomenon is more clearly demonstrated in EL than PL, either the electric field or the excitation of different energy levels plays a significant role. However, further study is needed to conclusively establish the mechanisms responsible for the observed phenomena.

To summarize, we have fabricated $\mathrm{Alq}_{3}$-based OLEDs with different device thickness values. For a range of devices, multiple peak emissions were observed, and the EL spectra exhibited significant variation with the increase of the viewing angle. With increasing viewing angle, energy exchange between the two peaks can be clearly resolved in the EL spectra, which has some similarities with the behavior of polariton PL. For optimized device thickness, near white emission with CIE coordinates $(0.32,0.43)$ can be obtained.

This work is supported by The Research Grants Council of the Hong Kong Special Administrative Region (Project No. HKU 7056/02E and HKBU 2051/02P). The authors would like to thank Dr. J. Gao for the use of step profiler to verify the device thickness.

${ }^{1}$ X. Zhou, M. Pfeiffer, J. S. Huang, J. Blochwitz-Nimoth, D. S. Qin, A. Werner, J. Drechsel, B. Maening, and K. Leo, Appl. Phys. Lett. 81, 922, (2002).

${ }^{2}$ Z. D. Popovic and H. Aziz, IEEE J. Sel. Top. Quantum Electron. 8, 362 (2002).

${ }^{3}$ Y. Kijima, N. Asai, and S-I. Tamura, Jpn. J. Appl. Phys., Part 1 38, 5274 (1999).

${ }^{4}$ T. Tsuji, S. Naka, H. Okada, and H. Onnagawa, Appl. Phys. Lett. 81, 3329 (2002).

${ }^{5}$ S. K. So, W. K. Choi, L. M. Leung, and K. Neyts, Appl. Phys. Lett. 74, 1939 (1999).

${ }^{6}$ P. E. Burrows, V. Khalfin, G. Gu, and S. R. Forrest, Appl. Phys. Lett. 73, 435 (1998)

${ }^{7}$ V. Bulović, V. B. Khalfin, G. Gu, P. E. Burrows, D. Z. Garbuzov, and S. R. Forrest, Phys. Rev. B 58, 3730 (1998).

${ }^{8}$ T. Shiga, H. Fujikawa, and Y. Taga, J. Appl. Phys. 93, 19 (2003).

${ }^{9}$ A. Dodabalapur, L. J. Rothberg, R. H. Jordan, T. M. Miller, R. E. Slusher, and J. M. Phillips, J. Appl. Phys. 80, 6954 (1996).

${ }^{10}$ S. Dirr, S. Wiese, H.-H. Johanns, D. Ammermann, A. Böhler, W. Grahn, and W. Kowalsky, Synth. Met. 91, 53 (1997).

${ }^{11}$ R. H. Jordan, L. J. Rothberg, A. Dodabalapur, and R. E. Slusher, Appl. Phys. Lett. 69, 1997 (1996).

${ }^{12}$ J. S. Kim, B. W. Seo, and H. B. Gu, Synth. Met. 132, 285 (2003).

${ }^{13}$ M. Mazzeo, D. Pisignano, L. Favaretto, G. Sotgiu, G. Barbarella, R. Cingolani, and G. Gigli, Synth. Met. 139, 675 (2003).

${ }^{14}$ R. J. Curry and W. P. Gillin, J. Appl. Phys. 88, 781 (2000).

${ }^{15}$ W. Humbs, H. Zhang, and M. Glasbeek, Chem. Phys. 254, 319 (2000).

${ }^{16}$ R. F. Oulton, N. Takada, J. Koe, P. N. Stavrinou, and D. D. C. Bradley, Semicond. Sci. Technol. 18, S419 (2003). 